\title{
O uso de ácido valproico para a indução de modelos animais de autismo: uma revisão
}

\author{
The use of valproic acid for the induction of \\ animal models of autism: a review \\ Eloise Schlickmann', Jucélia Jeremias Fortunato
}

\section{RESUMO}

Introdução: $\bigcirc$ autismo é um distúrbio neuropsiquiátrico com base biológica significativa. Faz parte de um grupo de condições definidas como transtornos invasivos do desenvolvimento e é caracterizado de um ponto de vista comportamental, com etiologias múltiplas e graus variados de gravidade. Entre os modelos animais de autismo estudados mais extensivamente estão aqueles derivados da exposição a agentes teratogênicos. Objetivo: $\bigcirc$ objetivo deste estudo foi mostrar evidências sobre a associação entre a exposição ao ácido valproico e o desenvolvimento de sinais clínicos semelhantes aos encontrados em pacientes autistas, por meio de uma revisão de literatura. Método: Este é um artigo de revisão de literatura realizado mediante pesquisas no MedLine e SciELO. Foram utilizadas as palavras-chave "autismo", "ácido valproico" e "modelo animal de autismo". Selecionaram-se artigos publicados

\section{Palavras-chave}

Autismo, modelo animal de autismo, ácido valproico.

\section{Keywords}

Autism, animal model of autism, valproic acid. entre os anos de 2008 a 2012. Resultados: Foi encontrado um total de 119 artigos e, desses, foram selecionados 22 artigos. Conclusão: Modelos animais gerados pela exposição de pré ou pós-natal de ácido valproico apresentam características adequadas para o estudo do autismo, principalmente porque evidenciam características comportamentais similares àquelas encontradas em indivíduos autistas.

\section{ABSTRACT}

Introduction: Autism is a neuropsychiatric disorder with significant biological basis. Part of a group of conditions defined as pervasive developmental disorders, and is characterized from a behavioral point of view, with multiple etiologies and varying degrees of severity. Among the animal models of autism studied most extensively are those derived from exposure to teratogenic agents. Objective: The The aim of this study was to provide evidence on the association between exposure to valproic acid and the development of clinical signs similar to those found in autistic patients, through a literature review. Method: This article is a literature review conducted by searching MedLine and SciELO. We used the keyword autism, valproic acid animal model of autism. We selected articles published between the years 2008 to 2012.

Results: We found a total of 119 articles were selected and these 22 articles. Conclusion: Animal models generated by exposure to prenatal or postnatal valproic acid have characteristics suitable for the study of autism, mainly because behavioral traits show similar to those found in autistic individuals.

1 Universidade do Sul de Santa Catarina (Unisul), Laboratório de Neurociências, Programa de Mestrado em Ciências da Saúde.

Endereço para correspondência: Jucélia Jeremias Fortunato

Av. José Acácio Moreira, 787 - Caixa Postal 370

88704-900 - Tubarão, SC, Brasil

Telefone: (55 48) 3621-3363

E-mail: jucelia.fortunato@unisul.br 


\section{INTRODUÇÃO}

O transtorno do espectro autista (TEA) é um distúrbio neuropsiquiátrico com base biológica significativa, que se desenvolve nos primeiros três anos de vida'. É parte de um grupo de condições definidas como transtornos invasivos do desenvolvimento (TID)2 , caracterizadas de um ponto de vista comportamental, com etiologias múltiplas e graus variados de gravidade ${ }^{3,4}$

Em busca de evidências que justificassem a precocidade das manifestações clínicas, os conceitos teóricos sobre a etiologia e a dinâmica desse transtorno se modificaram gradativamente ao longo do tempo. Inicialmente, o termo "autismo" foi utilizado por Leo Kanner $(1943)^{5}$ para descrever uma síndrome caracterizada pela incapacidade inata de estabelecer contato afetivo e interpessoal.

O diagnóstico e as subclassificações do autismo estiveram sob o amplo rótulo de esquizofrenia infantil por muitas décadas ${ }^{6}$. No entanto, Kanner acreditava que o autismo deveria ser separado da esquizofrenia, embora tivesse íntima relação com ela. Para ele, o isolamento social autista era diferente do evitamento social observado na esquizofrenia. Além disso, as crianças com autismo não desenvolviam alucinações, características muito comuns na esquizofrenia ${ }^{7}$, e mostravam sinais de perturbações muito antes das crianças com esquizofrenia manifestarem sintomatologia psicótica ${ }^{8,9}$.

Apesar de que diversos estudos clínicos tenham seguido após as descrições iniciais de Kanner, somente em 1980, com a publicação da terceira edição do Manual Diagnóstico e Estatístico de Transtornos Mentais (DSM-III), da Associação Americana de Psiquiatria (American Psychiatric Association, 1980), o autismo foi classificado como um tipo de transtorno invasivo do desenvolvimento e classificado como uma desordem distinta da esquizofrenia ${ }^{10}$.

Atualmente o diagnóstico é feito por avaliação clínica e requer a presença do distúrbio em três domínios: 1) interação social; 2) comunicação; 3) interesses restritos e padrões estereotipados do comportamento ${ }^{2,3,11}$. Se forem utilizados os critérios aceitos pela Organização Mundial de Saúde (CID, atualmente na décima edição) ${ }^{12}$ ou pela Associação Americana de Psiquiatria (DSM, atualmente na quarta edição) ${ }^{13}$, esse, certamente, não é um distúrbio raro³.

A incidência de TEA tem aumentado de forma significativa em todo o mundo ${ }^{14}$. O TEA ocupa o terceiro lugar entre os distúrbios do desenvolvimento, estando à frente das malformações congênitas e da síndrome de Down ${ }^{3}$. Afeta aproximadamente 1 em 150 crianças e adolescentes em vários países ${ }^{15}$, sendo mais comum em homens, numa proporção de 4:116.

Por causa da grande variabilidade de manifestações sintomatológicas, até o momento não foi possível determinar qualquer aspecto biológico, ambiental, ou da interação entre ambos, que contribua de forma decisiva para as manifestações do transtorno ${ }^{17,18}$.
O TEA apresenta um forte componente genético. A possibilidade de ocorrência do TEA em uma família que já possui uma criança autista é considerada elevada (de 3\% a 8\%)3. Estudos envolvendo irmãos gêmeos monozigóticos fornecem fortes evidências de hereditariedade genética ${ }^{19,20}$, sendo estimada em aproximadamente $60 \%{ }^{19}$, enquanto em gêmeos dizigóticos a taxa é de $0^{20,21}$. A reavaliação para características mais abrangentes, como déficits em comunicação e alterações sociais, faz com que essa concordância aumente de $60 \%$ para 92\% em monozigóticos e de 0\% para 10\% em dizigóticos ${ }^{22}$.

Extensas investigações revelaram o envolvimento de diferentes áreas encefálicas na gênese do TEA, incluindo estruturas do sistema límbico, amígdala, áreas pré-frontais e cerebelo ${ }^{14,15,23}$. As anormalidades poderiam ser induzidas em múltiplos períodos pré-natais, especialmente nas regiões cerebrais que se desenvolvem tardiamente na neurogênese. Além disso, regiões cerebrais envolvidas com o TEA tendem a se desenvolver mais lentamente e são mais vulneráveis a distúrbios ${ }^{24}$.

Os fatores ambientais parecem também estar envolvidos na alta prevalência do TEA. A influência ambiental inclui alterações fisiopatológicas do sistema nervoso central (SNC), incluindo estresse oxidativo, neuroinflamação, disfunção mitocondrial e distúrbios bioquímicos ${ }^{25}$. Evidências clínicas ${ }^{26}$ e experimentais ${ }^{27-30}$ mostram que infecções virais (tais como rubéola ${ }^{31}$ e citomagalovírus ${ }^{31,32}$ e bacterianas pré-natais ${ }^{28,33}$ igualmente podem interferir no ambiente intrauterino, promovendo anormalidades comportamentais com similaridades ao autismo ${ }^{27,28,31}$, inclusive com a ativação inadequada de citocinas pró-inflamatórias ${ }^{26,28,32}$ e alterações na resposta imune $e^{28,34}$.

Além disso, investigações mostram que a exposição a certos medicamentos como a talidomida e o ácido valproico (VPA) no período pré-natal e pós-natal pode alterar o desenvolvimento do cérebro e resultar em anormalidades comportamentais e déficits cognitivos ${ }^{42}$. O VPA é comercializado no mercado como um anticonvulsivante desde 1974 e é usado em muitos países por causa de sua eficiência contra vários tipos de epilepsia e como um estabilizador do humor. Uma das suas principais ações é o aumento no nível do ácido gama-aminobutírico (GABA) no cérebro43.

O VPA tem sido descrito como um agente teratogênico que promove anormalidades comportamentais e alterações em órgãos dos sistemas respiratório, cardiovascular, geniturinário, gastrintestinal, endócrino e esquelético ${ }^{37-39}$. Mulheres em idade fértil tratadas com esse fármaco devem usar contraceptivos e interromper a medicação antes de qualquer gravidez planejada. Se não houver nenhuma outra alternativa para o tratamento com VPA, a gravidez deve ser considerada de alto risco e deve ter o acompanhamento adequado, incluindo o diagnóstico pré-natal ${ }^{36}$. Logo após o VPA ter sido introduzido no mercado para uso clínico como anticonvulsivante, surgiram casos de alterações no tubo neural, 
particularmente de espinha bífida, entre os filhos expostos a esse medicamento no início de gestação ${ }^{40}$. Em humanos, a exposição ao VPA durante os dias 20-24 de gestação, ou seja, antes do fechamento do tubo neural, está relacionada à maior incidência de distúrbios de desenvolvimento neurológico, afetando principalmente as funções cognitiva e comportamental, semelhante àquelas encontradas no TEA ${ }^{41}$. De acordo com Rasalam, a taxa de TEA, incluindo transtorno invasivo do desenvolvimento e síndrome de Asperger, pode ser 20 vezes maior em crianças expostas ao ácido valproico do que a taxa esperada na população geral42. Essas alterações podem afetar o desenvolvimento da linguagem e de habilidades de aprendizagem ${ }^{36}$.

Uma vez que a fisiopatologia do TEA permanece desconhecida, sendo um transtorno complexo de grande heterogeneidade, o desenvolvimento de modelos animais refletindo aspectos comportamentais, neuropatológicos e genéticos é de extrema importância ${ }^{43}$. Para muitas doenças humanas, a geração e a caracterização de modelos animais são uma ponte essencial entre a compreensão das características moleculares da doença e o desenvolvimento de agentes terapêuticos ${ }^{44}$. Assim, modelos animais não humanos podem contribuir no entendimento das bases neurobiológicas desse transtorno ${ }^{45}$.

Modelos experimentais utilizando medicamentos são os modelos de TEA mais reproduzidos pelos pesquisadores e levam em conta os achados comportamentais, neurais, imunes e genéticos encontrados nos autistas ${ }^{28}$.

Dos agentes ambientais ligados ao TEA, o VPA é o que está sendo estudado mais extensivamente. Um dos modelos baseia-se na exposição de ratas prenhas ao ácido valproico no nono dia gestacional, afetando a migração de neurônios como os serotoninérgicos, ou no $12^{\circ}$ dia de gestação, podendo afetar a diferenciação neural. Essa exposição não produz anormalidades neuroanatômicas, no entanto os filhotes apresentam distúrbios comportamentais, como o aumento de tempo gasto em atividades estereotipadas semelhantes aos pacientes autistas ${ }^{46}$.

Para o TEA, assim como para outros TID, os modelos experimentais não humanos representam uma ferramenta importante para o entendimento das bases neuroanatômicas, moleculares e funcionais comprometidas. Considerando os diversos achados em diferentes modelos animais de TEA, O objetivo deste artigo é mostrar evidências sobre a associação entre a exposição ao VPA e o desenvolvimento de sinais clínicos semelhantes àqueles encontrados em pacientes autistas, por meio de uma revisão de literatura.

\section{MÉTODOS}

A pesquisa bibliográfica foi realizada por meio da busca eletrônica de artigos indexados nas bases de dados do
MedLine e SciELO, a partir das palavras-chave "autismo e ácido valproico" e "modelo animal de autismo e ácido valproico" e suas respectivas traduções no idioma inglês ("autism and valproic acid"; "animal model of autism and valproic acid"). A busca se restringiu a artigos publicados no período compreendido entre os anos de 2008 e 2012.

A amostra compreendeu publicações selecionadas da leitura prévia dos títulos e resumos indexados que respeitaram os seguintes critérios de inclusão: 1) periódicos indexados; 2 ) artigos publicados na íntegra em língua inglesa e portuguesa; 3) artigos publicados entre os anos de 2008 e 2012; 4) estudos nos quais as referências tiveram pertinência com o tema, tendo como critério principal os que abordavam o efeito teratogênico do VPA como fator determinante para a indução de modelo experimental de autismo.

Foram excluídos estudos publicados sob a forma de editoriais, entrevistas, notas clínicas, revisões e estudos com humanos, além daqueles que não descreveram a dosagem do VPA. Artigos que não atenderam a algum dos critérios propostos foram excluídos das análises posteriores.

\section{RESULTADOS}

Segundo os critérios de inclusão e exclusão desta revisão bibliográfica, foram encontrados 119 artigos, dos quais foram excluídos 97 estudos com base nos critérios apresentados, devendo-se considerar que: 1) desses, seis artigos se repetiam; 2) 12 eram artigos de revisão; 3) 62 eram estudos com humanos (estudo de caso, estudo transversal, entre outros); 4) nove dos artigos não descreveram a dosagem do VPA; 5) três estudos não se apresentavam na íntegra; 6) cinco artigos comparavam o efeito do VPA com os de outras substâncias. Finalmente, a revisão foi realizada com base em 22 artigos.

Os resultados apresentados a seguir se restringem às análises conduzidas com o objetivo de identificar o efeito teratogênico do VPA como fator determinante para a indução do modelo experimental de autismo.

Os artigos selecionados avaliaram de forma isolada os achados comportamentais, neurais, imunes e genéticos encontrados no modelo experimental de TEA, a partir da administração de VPA, em diferentes doses e vias, em períodos específicos da prenhez do animal.

Neste artigo de revisão foi encontrada uma série de estudos com roedores, relacionando o efeito teratogênico do VPA e o TEA. Os estudos referiram-se principalmente aos efeitos do VPA sobre o desenvolvimento do sistema nervoso central e incluem alterações morfológicas, bioquímicas e estudos comportamentais ${ }^{57-59}$.

A hipótese de que a exposição ao VPA consegue mimetizar sintomas comportamentais semelhantes àqueles encontrados em pacientes autistas foi descrita em todos os artigos selecionados. A maioria dos artigos selecionados (19) men- 
ciona os efeitos teratogênicos do ácido valproico a partir de sua administração em período pré-natal. Três artigos ${ }^{47,49,56}$ utilizaram especificamente o VPA no período pós-natal e apresentaram resultados semelhantes aos demais quando se referem às alterações neuroquímicas e comportamentais observadas no TEA.

Estudos mostram que durante o desenvolvimento precoce do cérebro machos e fêmeas respondem de forma diferente a agentes teratogênicos e substâncias tóxicas. Segundo os achados de Schneider et al.55, as funções comportamentais, imunológicas e endócrinas no autismo estão relacionadas ao gênero.

Considerando que pacientes autistas apresentam várias anormalidades no sistema imunológico, dois dos artigos selecionados ${ }^{5,64}$ mediram os parâmetros bioquímicos dos animais e encontraram alterações nos níveis basais de corticosterona e na produção de óxido nítrico, confirmando as semelhanças entre as anormalidades de roedores expostos ao VPA e a função imune em autistas.

\section{DISCUSSÃO}

Estudos em modelos animais fornecem grandes possibilidades de investigações que não são possíveis em humanos, incluindo análises imunológicas e do desenvolvimento, plasticidade e sinalização neural. O autismo é uma condição particularmente difícil de ser mimetizada em animais. A empatia e a capacidade de intuir os sentimentos e intenções de terceiros parecem ser impossíveis de ser medidas em ratos ou camundongos $^{69}$. Por outro lado, os roedores são, em geral, espécies que têm altos índices de interação e comunicação social ${ }^{70}$. Essa característica faz com que mudanças no comportamento social dos roedores sejam relacionadas a fatores ambientais ou genéticos ${ }^{70,71}$.

Entre os modelos que utilizam agentes teratogênicos como fatores de risco para o autismo, o VPA tem se mostrado como importante ferramenta para o estudo da etiologia desse transtorno ${ }^{11}$. Além disso, alterações neuromorfológicas $^{72,73}$ e neuroquímicas ${ }^{74}$ observadas em portadores dos TID

Tabela 1. Administração de VPA para caracterização de modelos experimentais de autismo. Descrições baseadas em trabalhos publicados entre os anos 2008 e 2012 e selecionados de acordo com as estratégias de busca. Os estudos estão organizados de acordo com a dose e via de administração utilizada e apresentados obedecendo à ordem cronológica da publicação

\begin{tabular}{|c|c|c|}
\hline $\begin{array}{l}\text { Dose e via de } \\
\text { administração } \\
\text { do VPA }\end{array}$ & Principal efeito do VPA & Referência \\
\hline \multirow[t]{5}{*}{$400 \mathrm{mg} / \mathrm{kg}$, s.c. } & Aumento de células apoptóticas hipocampais e cerebelares & Yochum et al. (2010) \\
\hline & Padrões comportamentais anormais, especialmente quando expostos ao VPA no $12^{\circ}$ dia gestacional & Kim et al. (2011) \\
\hline & Formação de marcadores de estresse oxidativo que foram revertidos pela administração crônica de extrato de chá verde & Banji et al. (2011) \\
\hline & Padrões comportamentais anormais que foram revertidos pela administração crônica de ginseng vermelho coreano & Kimetal. (2012) \\
\hline & Aumento na expressão das proteínas Wnt e proliferação de células progenitoras neurais & Goet al. (2012) \\
\hline \multirow[t]{3}{*}{$500 \mathrm{mg} / \mathrm{kg}$, i.p. } & Diminuição da excitabilidade intrínseca de neurônios piramidais & Rinaldi et al. (2008) \\
\hline & Hiper-reatividade da amígdala associada ao seu envolvimento na consolidação da memória do medo & Markram et al. (2008) \\
\hline & Redução do número de células no córtex pré-frontal, mas não no córtex somatossensorial de fêmeas & Hara et al. (2012) \\
\hline \multirow[t]{8}{*}{$600 \mathrm{mg} / \mathrm{kg}$, i.p. } & Padrões comportamentais anormais e função imunológica alterada & Schneider et al. (2008) \\
\hline & Aumento da atividade locomotora e do número de células cerebelares e hipocampais & Yochum et al. (2008) \\
\hline & Aquisição precoce e maior magnitude de resposta condicionada & Murawski et al. (2009) \\
\hline & Aumento na expressão das proteínas Wnt & Wang et al. (2010) \\
\hline & Exerce ação direta e indireta sobre o sistema serotoninérgico & $\begin{array}{l}\text { Dufour-Rainfray et al. } \\
\text { (2010) }\end{array}$ \\
\hline & $\begin{array}{l}\text { Aumento da atividade sináptica no córtex pré-frontal medial observada nos modelos de facilitação por pulso pareado (PPF) e indução e } \\
\text { manutenção da potenciação de longa duração (LTP) }\end{array}$ & Sui et al. (2012) \\
\hline & $\begin{array}{l}\text { Padrões comportamentais anormais, formação de marcadores de estresse oxidativo e histoarquitetura cerebelar alterada. Resultados } \\
\text { revertidos pela administração do extrato de Bacopa monniera }\end{array}$ & Sandhya et al. (2012) \\
\hline & Padrões anormais de comportamento que foram revertidos pela administração de MPEP (um antagonista de receptor de glutamato) & Mehta et al. (2011) \\
\hline \multirow[t]{6}{*}{$800 \mathrm{mg} / \mathrm{kg}, \mathrm{v} .0$} & Hiperatividade da transmissão dopaminérgica associada à tarefa de natação forçada & Nakasato et al. (2008) \\
\hline & Migração anormal de neurônios TH-positivos e neurônios serotoninérgicos & Kuwagata et al. (2009) \\
\hline & Baixos níveis de expressão de RNAm da NLGN3 (neuroligina) no hipocampo, giro denteado e córtex somatossensorial & Kolozsi et al. (2009) \\
\hline & Padrões comportamentais anormais & Narita et al. (2010) \\
\hline & Atraso no desenvolvimento físico. Padrões comportamentais anormais. Baixos níveis de expressão cortical de RNAm para BDNF & Roullet et al. (2010) \\
\hline & Desenvolvimento anormal e imunorreatividade reduzida de nervos cranianos & Tashiro et al. (2011) \\
\hline
\end{tabular}

VPA: ácido valproico; s.c.: subcutâneo; i.c.v.: intracerebroventricular; i.p.: intraperitoneal; v.0.: oral. 
já foram demonstradas na prole de ratos e camundongos expostos ao VPA em período pré ou pós-natal ${ }^{11,42}$.

O desenvolvimento normal de um embrião é dependente de dois fenômenos principais: (a) instruções genéticas para a morfogênese e (b) capacidade dos tecidos para completar o processo metabólico geral, o que requer que as células sobrevivam e repliquem-se ${ }^{75}$. O SNC, comparado aos demais sistemas, apresenta sensibilidade mais prematura, com maior vulnerabilidade para doenças do tubo neural. As falhas do fechamento do tubo neural atrapalham a diferenciação do SNC, podendo resultar em anomalias do desenvolvimento ${ }^{76}$, incluindo o autismo.

Nesse sentido, os modelos animais que utilizam a exposição de VPA para promover anormalidades comportamentais e alterações sistêmicas similares àquelas encontradas em indivíduos autistas ${ }^{37,77}$ apontam seu efeito neurotóxico preferencialmente quando ele é administrado na primeira metade da gestação de ratos (grande parte dos estudos aponta para o período compreendido entre os dias 9 e 14, após confirmação da prenhez). Nesse período, o VPA interfere no metabolismo do ácido fólico e aumenta o risco de malformações do tubo neural|78,79.

A investigação dos possíveis mecanismos pelos quais os teratógenos podem levar à manifestação do TEA foi direcionada para as alterações no sistema serotoninérgico, como mudança dos neurônios 5-HT e anormalidades na formação deles como resultado da exposição ao VPA durante o período crítico da embriogênese dos roedores ${ }^{73}$. Além disso, estudos feitos nas últimas décadas nos cérebros de autistas revelaram alterações na anatomia de regiões cerebrais ${ }^{72}$, sugerindo que a origem do TEA ocorra ainda na vida pré-natal.

Esses resultados permitem caracterizar a exposição ao VPA como um modelo adequado para o estudo do autismo, uma vez que os animais submetidos sistematicamente a esse agente teratogênico conseguiram estabelecer as três características principais de um modelo animal de doença: (a) validade de face; (b) validade de constructo e (c) validade preditiva $^{80}$.

\section{CONCLUSÃO}

Em razão da multiplicidade etiológica e fenotípica do TEA, esse parece ser resultado de uma complexa combinação de fatores ambientais, neurológicos, imunológicos e genéticos. A compreensão da neuropatologia do TEA tem avançado substancialmente ao longo dos últimos anos, mas ainda há muitas questões que permanecem sem solução.

Apesar de haver algumas limitações inerentes ao estudo de revisão, tais como a diversidade dos modelos propostos, especialmente quando se refere às diferenças de gênero e idade dos animais experimentais, ou, ainda, conclusões restritas aos trabalhos publicados no período considerado para este estudo, cada um dos modelos mencionados parece capturar pelo menos uma das peças do espectro autista. Além disso, a reprodutibilidade dos modelos apresentados, especialmente daqueles que tratam de análises comportamentais, apresenta semelhanças reais com estudos clínicos, considerando que o diagnóstico desse transtorno é dado basicamente por meio da análise comportamental.

Nesse sentido, estudos com modelos animais e ensaios clínicos devem manter sintonia, a fim de possibilitar o entendimento sobre o quão segura e eficazmente os dados de roedores podem predizer as respostas em humanos. É essencial a colaboração interdisciplinar entre pesquisadores para garantir sucesso e viabilizar estratégias de reversão das consequências em longo prazo, e não apenas controle dos sintomas das desordens neurobiológicas. E, ainda, aumentar a interação entre a pesquisa básica e clínica para que os estudos controlados em humanos, bem como em modelos animais, possam, em associação, promover melhor qualidade de vida para pessoas com transtornos neuropsiquiátricos, incluindo o autismo.

\section{CONTRIBUIÇÕES INDIVIDUAIS}

\section{Eloise Schlickmann e Jucélia Jeremias Fortunato -} Contribuíram significativamente na concepção do estudo e substancialmente na elaboração do artigo e na revisão crítica do seu conteúdo intelectual. Participaram na aprovação da versão final a ser publicada.

\section{CONFLITOS DE INTERESSES}

Os autores não possuem conflitos de interesse a serem declarados.

\section{AGRADECIMENTOS}

O presente estudo compreende parte de um projeto financiado pela Fundação de Amparo à Pesquisa e Inovação do Estado de Santa Catarina (Fapesc) e Universidade do Sul de Santa Catarina (Unisul). As autoras agradecem a essas instituições.

\section{REFERÊNCIAS}

1. Schneider T, Przewlocki R. Behavioral alterations in rats prenatally exposed to valproic acid: animal model of autism. Neuropsychopharmacol. 2005;30(1):80-9.

2. Nikolov R, Jonker J, Scahill L. Autismo: tratamentos psicofarmacológicos eáreas de interesse para desenvolvimentos futuros. Rev Bras Psiquiatr. 2006;28(1):39-46.

3. Gadia CA, Tuchman R, Rotta NT. Autism and pervasive developmental disorders. J Pediatr. 2004;80:83-94. 
4. Klin A. Autism and Asperger syndrome: an overview. Rev Bras Psiquiatr. 2006;28:3-12.

5. Kanner L. Autistic disturbances of affective contact. Nervous Child. 1943;2:217-50.

6. Tamanaha AC, Perissinoto J, Chiari BM. A brief historic review of the conceptions of Autism and Asperger syndrome. Rev Soc Bras Fonoaudiol. 2008;13(3):296-9.

7. Rumsey JM, Andreasen NC, Rapoport JL. Thought, language, communication, and affective flattening in autistic adults. Arch Gen Psychiatry. 1986;43:771-7.

8. DeLisi LE. The significance of age of onset for schizophrenia. Schizophr Bull. 1992;18: 209-15.

9. Goldstein G, Minshew NJ, Allen DN, Seaton BE. High-functioning autism and schizophrenia: a comparison of an early and late onset neurodevelopmental disorder. Arch Clin Neuropsychol. 2002;17(5):461-75.

10. Sugranyes G, Kyriakopoulos M, Corrigall R, Taylor E, Frangou S. Autism spectrum disorders and schizophrenia: meta-analysis of the neural correlates of social cognition. PLOS One. 2011;6(10):1-13.

11. Bambini-Junior V, Rodrigues L, Behr GA, Moreira JC, Riesgo R, Gottfried C. Animal model of autism induced by prenatal exposure to valproate: behavioral changes and liver parameters. Brain Res. 2011;23:1408-8.

12. Classificação dos transtornos mentais e de comportamento da CID10: descrições clínicas e diretrizes diagnósticas. Porto Alegre: Artes Médicas; 1993.

13. American Psychiatry Association. Diagnostic and statistical manual of mental disorders. 4th ed. Washington, DC; 2000

14. Schroeder JH, Desrocher M, Bebko JM, Cappadocia MC. The neurobiology of autism: theoretical applications. Res Autism Spectr Disord. 2010;4(4):555-64.

15. Rossignol DA, Bradstreet JJ. Evidence of mitochondrial dysfunction in autism and implications for treatment. Am J Biochem Biotechnol. 2008:4:208-17.

16. Theoharides TC, Kempuraj D, Redwood L. Autism: an emerging 'neuroimmune disorder' in search of therapy. Expert Opin Pharmacother. 2009;10:127-43.

17. Silva M, Mulick JA. Diagnosticando o transtorno autista: aspectos fundamentais e considerações práticas. Psicol Cienc Prof. 2009;29(1):116-31.

18. Rivet TT, Matson JL. Review of gender differences in core symptomatology in autism spectrum disorders. Res Autism Spectr Disord. 2011;5:957-76.

19. Freitag CM. The genetics of autistic disorders and its clinical relevance: a review of the literature. Mol Psychiatry. 2007;12(1):2-22.

20. Brkanac Z, Raskind WH, King BH. Pharmacology and genetics of autism: implications for diagnosis and treatment. Per Med. 2008;5(6):599-607.

21. Bailey A, Le Couteur A, Gottesman I, Bolton P, Simonoff E, Yuzda E, et al. Autism as a strongly genetic disorder: evidence from a British twin study. Psychol Med. 1995;25:63-77.

22. Casanova MF. Neuropathological and genetic findings in autism: the significance of a putative minicolumnopathy. Neuroscientist. 2006;12(5):435-41.

23. Bugalho P, Correa B, Viana-Baptista M. Role of the cerebellum in cognitive and behavioural control: scientific basis and investigation models. Acta Med Port. 2006;19:257-67.

24. Ashwood P, Wills $S$, Van de Water J. The immune response in autism: a new frontier for autism research. J Leukoc Biol. 2006;80(1):1-15

25. Herbert MR. Contributions of the environment and environmentally vulnerable physiology to autism spectrum disorders. Curr Opin Neurol. 2010;23:103-10.

26. Atladóttir HO, Thorsen P, Ostergaard L, Schendel DE, Lemcke S, Abdallah M, et al. Maternal infection requiring hospitalization during pregnancy and autism spectrum disorders. J Autism Dev Disord. 2010;40(12):1423-30.

27. Shi L, Fatemi SH, Sidwell RW, Patterson PH. Maternal influenza infection causes marked behavioral and pharmacological changes in the offspring. J Neurosci. 2003;23(1):297-302.

28. Bernardi MM, Kirsten TB, Trindade MO. Sobre 0 autismo, neuroinflamação e modelos animais para o estudo de autismo: uma revisão. Rev Neurocienc. 2012;20(1):117-27.

29. Boksa P. Effects of prenatal infection on brain development and behavior: a review of findings from animal models. Brain Behav Immun. 2010;24(6):881-97.

30. Hornig M, Weissenbock H, Horscroft N, Lipkin WI. An infection-based model of neurodevelopmental damage. Proc Natl Acad Sci U S A. 1999;96:12102-7.

31. Libbey JE, Sweeten TL, McMahon WM, Fujinami RS. Autistic disorder and viral infections. J Neurovirol. 2005:11(1):1-10.
32. Yamashita Y, Fujimoto C, Nakajima E, Isagai T, Matsuishi T. Possible association between congenital cytomegalovirus infection and autistic disorder. J Autism Dev Disord. 2003:33:455-9.

33. Kirsten TB, Taricano M, Maiorka PC, Palermo-Neto J, Bernardi MM. Prenatal lipopolysaccharide reduces social behavior in male offspring. Neuroimmunomodulation. 2010;17:240-51.

34. Dalton P, Deacon R, Blamire A, Pike M, McKinlay I, Stein J, et al. Maternal neuronal antibodies associated with autism and a language disorder. Ann Neurol. 2003:53:533-7.

35. Landrigan PJ. What causes autism? Exploring the environmental contribution. Curr Opin Pediatr. 2010;22:219-25.

36. Ornoy A. Valproic acid in pregnancy: how much are we endangering the embryo and fetus? Reprod Toxicol. 2009;28(1):1-10.

37. Robert E, Rosa F. Valproate and birth defects. Lancet. 1983;2(8359):1142.

38. DiLiberti J, Farndon P, Dennis N, Curry C. The fetal valproate syndrome. Am J Med Genet. 1984;19(3):473-81.

39. Alsdorf R, Wyszynski DF. Teratogenicity of sodium valproate. Expert Opin Drug Saf. 2005:4(2):345-53

40. Koren G, Nava-Ocampo AA, Moretti ME, et al. Major malformations with valproic acid. Can Fam Physician. 2006;52:444-7.

41. Rice DS, Barone Jr. Critical periods of vulnerability for the developing nervous system: evidence from humans and animal models. Environ Health Perspect. 2000;3:511-33.

42. Rasalam A, Hailey H, Williams J, Moore S, Turnpenny P, Lloyd D, et al. Characteristics of fetal anticonvulsant syndrome associated autistic disorder. Dev Med Child Neurol. 2005;47(8):551-5.

43. Cheh MA, Millonig JH, Roselli LM, Ming X, Jacobsen E, Kamdar S, et al. En2 knockout mice display neurobehavioral and neurochemical alterations relevant to autism spectrum disorder. Brain Res. 2006;1116(1):166-76.

44. LeBlanc JJ, Fagiolini M. Autism: a "critical period" disorder? Neural Plast. 2011;921680:1-17.

45. Lim MM, Bielsky IF, Young LJ. Neuropeptides and the social brain: potential rodent models in autism. Inter J Develop Neurosci. 2005;23:235-43.

46. Schneider T, Turczak J, Przewocki R. Environmental enrichment reverses behavioral alterations in rats prenatally exposed to valproic acid: issues for a therapeutic approach in autism. Neuropsychopharmacol. 2006;31:36-46.

47. Yochum CL, Bhattacharya P, Patti L, Mirochnitchenko 0, Wagner GC. Animal model of autism using GSTM1 knockout mice and early post-natal sodium valproate treatment. Behav Brain Res. 2010;210(2):202-10.

48. Kim KC, Kim P, Go HS, Choi CS, Yang SI, Cheong JH, et al. The critical period of valproate exposure to induce autistic symptoms in Sprague-Dawley rats. Toxicol Lett. 2011;201(2):137-42.

49. Banji D, Banji OJ, Abbagoni S, Hayath MS, Kambam S, Chiluka VL. Amelioration of behavioral aberrations and oxidative markers by green tea extract in valproate induced autism in animals. Brain Res. 2011;1410:141-51.

50. Kim P, Park JH, Kwon KJ, Kim KC, Kim HJ, Lee JM, et al. Effects of Korean red ginseng extracts on neural tube defects and impairment of social interaction induced by prenatal exposure to valproic acid. Food Chem Toxicol. 2012;51:288-96.

51. Go HS, Kim KC, Choi CS, Jeon SJ, Kwon KJ, Han SH, et al. Prenatal exposure to valproic acid increases the neural progenitor cell pool and induces macrocephaly in rat brain via a mechanism involving the GSK-3ß/B-catenin pathway. Neuropharmacol. 2012;63(6):1028-41.

52. Rinaldi T, Silberberg G, Markram H. Hyperconnectivity of local neocortical microcircuitry induced by prenatal exposure to valproic acid. Cereb Cortex. 2008;18(4):763-70.

53. Markram K, Rinaldi T, La Mendola D, SandiC, Markram H. Abnormal fear conditioning and amygdala processing in an animal model of autism. Neuropsychopharmacol. 2008;33(4):901-12

54. Hara Y, Maeda Y, Kataoka S, Ago Y, Takuma K, Matsuda T. Effect of prenatal valproic acid exposure on cortical morphology in female mice. J Pharmacol Sci. 2012;118(4):543-6.

55. Schneider T, Roman A, Basta-Kaim A, Kubera M, Budziszewska B, Schneider K, et al. Gender-specific behavioral and immunological alterations in an animal model of autism induced by prenatal exposure to valproic acid. Psychoneuroendocrinol. 2008;33(6):728-40.

56. Yochum CL, Dowling P, Reuhl KR, Wagner GC, Ming X. VPA-induced apoptosis and behavioral deficits in neonatal mice. Brain Res. 2008:1203:126-32.

57. Murawski NJ, Brown KL, Stanton ME. Interstimulus interval (ISI) discrimination of the conditioned eyeblink response in a rodent model of autism. Behav Brain Res. 2009;196(2):297-303. 
58. Wang Z, Xu L, Zhu X, Cui W, Sun Y, Nishijo H, et al. Demethylation of specific Wnt/B-catenin pathway genes and its upregulation in rat brain induced by prenatal valproate exposure. Anat Rec (Hoboken). 2010;293(11):1947-53.

59. Dufour-Rainfray D, Vourc'h P, Le Guisquet AM, Garreau L, Ternant D, Bodard S, et al. Behavior and serotonergic disorders in rats exposed prenatally to valproate: a model for autism. Neurosci Lett. 2010;470(1):55-9.

60. Sui L, Chen M. Prenatal exposure to valproic acid enhances synaptic plasticity in the medial prefrontal cortex and fear memories. Brain Res Bull. 2012;87(6):556-63.

61. Sandhya T, Sowjanya J, Veeresh B. Bacopa monniera (L.) Wettst ameliorates behavioral alterations and oxidative markers in sodium valproate induced autism in rats. Neurochem Res. 2012;37(5):1121-31.

62. Mehta MV, Gandal MJ, Siegel SJ. mGluR5-antagonist mediated reversal of elevated stereotyped, repetitive behaviors in the VPA model of autism. PLOS ONE. 2011;6(10):e26077.

63. Nakasato A, Nakatani Y, Seki Y, Tsujino N, Umino M, Arita H. Swim stress exaggerates the hyperactive mesocortical dopamine system in a rodent model of autism. Brain Res. 2008;: :128-35.

64. Kuwagata M, Ogawa T, Shioda S, Nagata T. Observation of fetal brain in a rat valproate-induced autism model: a developmental neurotoxicity study. Int J Dev Neurosci. 2009;27(4):399-405.

65. Kolozsi E, Mackenzie RN, Roullet Fl, deCatanzaro D, Foster JA. Prenatal exposure to valproic acid leads to reduced expression of synaptic adhesion molecule neuroligin 3 in mice. Neuroscience. 2009;163(4):1201-10.

66. Narita M, Oyabu A, Imura Y, Kamada N, Yokoyama T, Tano K, et al. Nonexploratory movement and behavioral alterations in a thalidomide or valproic acid-induced autism model rat. Neurosci Res. 2010;66(1):2-6.

67. Roullet Fl, Wollaston L, Decatanzaro D, Foster JA. Behavioral and molecular chances in the mouse in response to prenatal exposure to the anti-epileptic drug valproic acid. Neuroscience. 2010;170(2):514-22.

68. Tashiro Y, Oyabu A, Imura Y, Uchida A, Narita N, Narita M. Morphological abnormalities of embryonic cranial nerves after in utero exposure to valproic acid: implications for the pathogenesis of autism with multiple developmental anomalies. Int J Dev Neurosci. 2011;29(4):359-64

69. Miller G. Animal behavior. Signs of empathy seen in mice. Science. 2006;312(5782):1860-1.

70. Crawley JN. Behavioral phenotyping of rodents. Comp Med. 2003;53(2):140-6.

71. Crawley JN. Mouse behavioral assays relevant to the symptoms of autism. Brain Pathol. 2007;17(4):448-59.

72. Ingram JL, Peckham SM, Tisdale B, Rodier PM. Prenatal exposure of rats to valproic acid reproduces the cerebellar anomalies associated with autism. Neurotoxicol Teratol. 2000;22:319-24.

73. Miyazaki K, Narita N, Narita M. Maternal administration of thalidomide or valproico acid causes abnormal serotonergic neurons in the offspring: implication for pathogenesis of autism. Int J Dev Neurosci. 2005;23:287-97.

74. Tsujino N, Nakatani Y, Seki Y, Nakasato A, Nakamura M, Sugawara M, et al. Abnormality of circadian rhythm accompanied by an increase in frontal cortex serotonin in animal model of autism. Neurosci Res. 2007;57(2):289-95.

75. Poswillo D. Mechanisms and pathogenesis of malformation. Br Med Bull. 1976;32:59-64.

76. Elwood JM, Little J, Elwood JH. Epidemiology and control of neural tube defects. New York: Oxford University Press; 1992.

77. DiLiberti J, Farndon P, Dennis N, Curry C. The fetal valproate syndrome. Am J Med Genet. 1984;19(3):473-81.

78. Emmanouil-Nikoloussi EN, Foroglou NG, Kerameos-foroglou CH, Thliveris JA. Effects of valproic acid on fetal and maternal organs in the mouse: a morphological study. Morphologie. 2004;88:41-5.

79. Vorhees CV. Behavioral teratogenicity of valproic acid: selective effects on behavior after prenatal exposure to rats. Psychopharmacology. 1987;92:173-9.

80. Ellenbroek BA, Cools AR. Animal models with construct validity for schizophrenia. Behav Pharmacol. 1990;1(6):469-90. 